\title{
Surface Photovoltage Spectroscopy and AFM Analysis of CIGSe Thin Film Solar Cells
}

\author{
Nima E. Gorji, Ugo Reggiani, and Leonardo Sandrolini \\ Department of Electrical, Electronic, and Information Engineering, University of Bologna, 40136 Bologna, Italy
}

Correspondence should be addressed to Nima E. Gorji; nima.eshaghigorji2@unibo.it

Received 31 July 2014; Revised 17 September 2014; Accepted 28 October 2014

Academic Editor: Olindo Isabella

Copyright (C) 2015 Nima E. Gorji et al. This is an open access article distributed under the Creative Commons Attribution License, which permits unrestricted use, distribution, and reproduction in any medium, provided the original work is properly cited.

The band gap, grain size, and topography of a $\mathrm{Cu}(\mathrm{In}, \mathrm{Ga}) \mathrm{Se}_{2}$ (CIGSe) thin film solar cell are analyzed using surface photovoltage spectroscopy (SPV) and atomic force microscopy (AFM) techniques. From the steep increase in SPV signal the band gap of the CIGSe absorber, $\mathrm{In}_{2} \mathrm{~S}_{3}$ and $\mathrm{ZnO}$ layers are extracted and found to be 1.1, 1.3 and $2.6 \mathrm{eV}$, respectively. Already below the band gap of $\mathrm{ZnO}$ layer, a slight SPV response at $1.40 \mathrm{eV}$ photon energies is observed indicating the presence of deep donor states. The root mean square (rms) of the surface roughness is found to be $37.8 \mathrm{~nm}$ from AFM surface topography maps. The grain sizes are almost uniform and smaller than $1 \mu \mathrm{m}$.

\section{Introduction}

Surface photovoltage spectroscopy is a nondestructive but very precise technique to extract the band gap of thin film devices as well as the energy levels of shallow or deep defects distributed through the surface and bulk of the material [1]. Several different graded band gap profiles were proposed and simulated for CIGSe thin film solar cells [2]. Thus, the SPV technique could be used to extract the band gap of fabricated devices with similar graded profiles. Moreover, this technique can characterize the effect of grain boundaries and surface electronics which are the critical aspects of such polycrystalline materials [3]. CdTe semiconductors were already analyzed with the SPV method in order to verify the role of the level and concentration of deep defects on the electrical resistivity (or conductivity) of the layer after deposition [4]. It is well-known that the deep defects around the Fermi level or at the mid-gap $(0.7-0.8 \mathrm{eV})$ are responsible for the low conductivity of the semiconductor. In thin film solar cell fabrication, the postgrowth treatment and annealing at the high temperature can induce such defects across the film thickness. These recombination centers are the complexes of vacancy-ions or metallic defects interdiffused into the junction at high temperature. Moreover, the metallic back contacts can critically influence the carrier transport through the film. When uncompensated defects increase at the space charge region, the electric field is reduced to a narrower region at the junction. This causes an uncompleted transport of the carriers to the respective electrodes and increases the recombination or series resistance of the device. However, the SPV method has to be accompanied with other techniques which can detect the origin of such defects. Thus, investigating the grain boundaries, traps, and dislocations (morphology) of the films can assist to understand the defect/trap resources many of which diffuse from the back contact to the junction through the grain boundaries. Further studies on the surface topography of thin film devices can be achieved in nanometer scale by atomic force microscopy. AFM can scan the surface roughness and grain sizes with different orientation of the crystal facets which form areas with different work functions [1]. In the present study, the band gap, grain size, and topography of a CIGSe thin film device are analyzed using SPV and AFM techniques. The SPV signals for a wide range of illuminated photon energies can verify the graded band gap where the energy gap of each layer is extracted. Similar to photoluminescence studies, the amplitude of the peaks represents the presence of defects across the film. These defects are detected and scanned by the AFM method to analyze their origin and type. AFM analysis of Liao et al. on a similar device revealed that the film 
homogeneity, grain size, and surface roughness play a critical role on the characteristics of the finished device [5].

\section{Experimental Procedure}

The sample has been fabricated by a three-stage coevaporation process in the Institut des Matériaux Jean Rouxel, Université de Nantes, Nantes, France. The following data were provided by the manufacturer. The thin film solar cell investigated in this work is a heterojunction SLG/Mo/CIGSe/ $\mathrm{In}_{2} \mathrm{~S}_{3}$ / $\mathrm{ZnO} / \mathrm{ZnO}$ :Al. SLG is $3 \mathrm{~mm}$ soda-lime glass substrate coated with a $300 \mathrm{~nm}$ Mo layer. The thicknesses of CIGSe and $\mathrm{In}_{2} \mathrm{~S}_{3}$ are 1.8 and $50 \mu \mathrm{m}$, respectively. The $\mathrm{In}_{2} \mathrm{~S}_{3}$ buffer layer was grown by coevaporation of In and $\mathrm{S}$ elements. The ratio of In and $\mathrm{Ga}$ (group III) is $\mathrm{Ga} /(\mathrm{In}+\mathrm{Ga})=0.25$. The process was bithermal for the CIGSe (with chalcopyrite structure), $400-620^{\circ} \mathrm{C}$ measured by thermocouple at the back of the cell. The deposition temperature for $\operatorname{In}_{2} \mathrm{~S}_{3}$ was $175^{\circ} \mathrm{C}$. CIGSe has a chalcopyrite structure, and $\operatorname{In}_{2} S_{3}$ is in the beta form, which is a defect spinel structure. Further information about the fabrication details can be found in [6]. The band gap of the absorber layer should have been graded through a three-stage coevaporation process (between $1.1 \mathrm{eV}$ and $1.3 \mathrm{eV}$ ) and is $\mathrm{V}$-shape. The $\mathrm{Ga} /(\mathrm{In}+\mathrm{Ga})$ ratio rules the band gap and the "natural" gradients (inherent to the 3-stage process used for the CIGSe growth). The minimum Ga content (thus minimum $E_{g}$ ) is about $200 \mathrm{~nm}$ far from the CIGSe surface. In principal, the minimum $E_{g}$ is about $1.1 \mathrm{eV}$ whereas the top CIGSe as well as the CIGSe close to the Mo back contact can be $1.2-1.3 \mathrm{eV}$. The area of the cell is $0.5 \mathrm{~cm}^{2}$.

Then, the CIGSe/ $/ \operatorname{In}_{2} \mathrm{~S}_{3}$ structure has been covered with standard window bilayer consisting of $\mathrm{ZnO}(50 \mathrm{~nm})$ and conductive $\mathrm{ZnO}$ :Al $(200 \mathrm{~nm})$. These two oxide layers have been deposited consecutively in the same chamber by RF sputtering (no air exposure after the $\mathrm{ZnO}$ deposition). $\mathrm{CIGSe} / \mathrm{In}_{2} \mathrm{~S}_{3}$ structure has been rinsed with deionized water. Then the completed device was annealed in air at $200^{\circ} \mathrm{C}$ for 20 minutes.

For the SPV measurements at room temperature, the photon beam generated by a white light source passed through the monochromator SPEX 500M and was focused onto the sample surface. The contribution of the local photocurrent to the tunneling current while sweeping the voltage applied to the conductive tip allowed the measurement of the SPV signal in a wide spectral range from 300 to $2000 \mathrm{~nm}$ using different gratings and light sources (quartz-tungsten-halogen and xenon lamps). In the SPV experiment, with the beam on, at the interface of the CIGSe/ $\operatorname{In}_{2} \mathrm{~S}_{3}$ layer the equilibrium was driven away and the photons of the beam created the electron hole pairs. The built-in potential separates the pairs near the band edge which creates a negative photovoltage at the CIGSe surface called surface photovoltage. However, the other properties both from surface and subsurface of the film, that is, the width of the surface space charge region and the recombination rate, contributed to the SPV signal [7-9]. Moreover, surface topography was performed by noncontact mode AFM (NT MDT-Solver PRO 47). Silicon tips coated with $\mathrm{Pt} / \mathrm{Ir}$, diamond tips and conductive nanoneedles $\left(\mathrm{Ag}_{2} \mathrm{Ga}\right.$

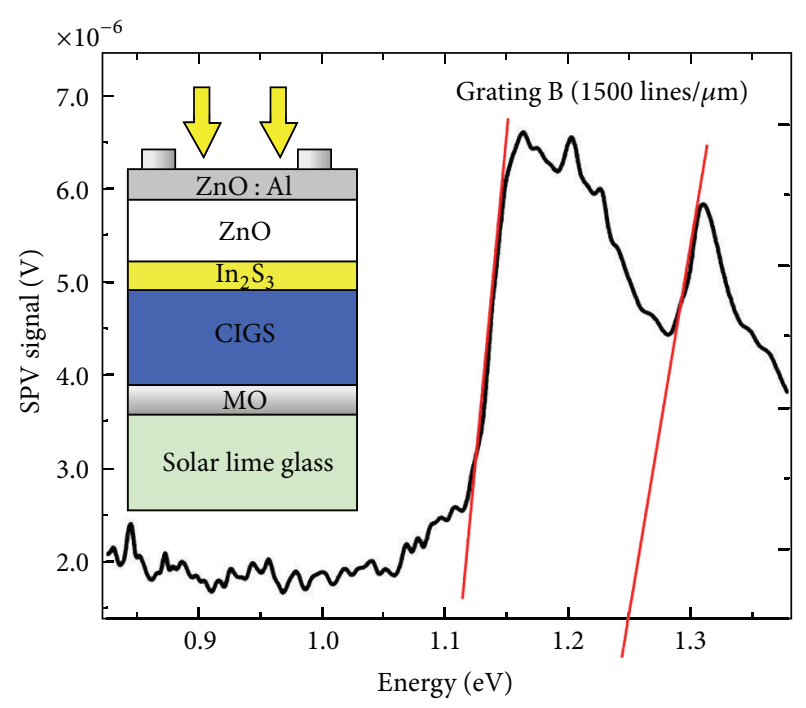

FIGURE 1: SPV signal versus photon energy impinging through grating B (1500 lines/ $\mu \mathrm{m})$.

Nauganeedles) were used to obtain the current maps $\left(\mathrm{Ag}_{2} \mathrm{Ga}\right.$ Nauganeedles). The topographic and current images were obtained simultaneously, as AFM tip scans the surface and the current is measured. SPV and AFM analyses were carried out in the laboratory "Physics of Defects in Semiconductors," Department of Physics, University of Bologna, Bologna, Italy.

\section{Results and Discussion}

3.1. SPV Analysis. Surface photovoltage spectroscopy analysis is based on measuring the SPV signal (which is a lightinduced variation of the surface potential) as a function of the impinging photon beam energy. Here, we consider the application of SPV measurements on CIGSe materials for the approximate determination of the graded band gap and understanding the surface formation.

Figure 1 shows the SPV signal plotted versus the energy of impinging photons passing through grating B with 1500 lines $/ \mu \mathrm{m}$ for photon energies in the range of $0.8-1.4 \mathrm{eV}$. The most important features to interpret a SPV spectrum are the sharp slope changes, "knees", associated with an abrupt increase of an additional carrier excitation mechanism. At low photon excitation energies (below $0.8 \mathrm{eV}$ ) the SPV signal is undetectable since the light energy is not sufficient to excite the charge carriers in CIGSe material. Three peaks and two knees for $h v>1.1 \mathrm{eV}$ are displayed in Figure 1. At around 1.1 and $1.2 \mathrm{eV}$, two peaks are observed with almost the same amplitude of $6.5 \mu \mathrm{V}$. The next peak is seen at energies higher than $1.3 \mathrm{eV}$ but with a lower SPV signal magnitude $(5.9 \mu \mathrm{V})$. These results indicate that the $E_{g}$ of CIGSe is graded with a V-shape with the minimum of $1.1 \mathrm{eV}$ whereas it is about $1.2-1.3 \mathrm{eV}$ at the top and close to Mo. We might also note that the peak related to $1.3 \mathrm{eV}$ can be due to $\mathrm{Cu}$ interdiffusion to $\mathrm{In}_{2} \mathrm{~S}_{3}$ (formation of $\operatorname{In}_{2} \mathrm{~S}_{3}: \mathrm{Cu}$ ). Furthermore, the first knee occurs at subband gap photon energies and corresponds to the onset of carrier excitation from surface 
states. This assumption should also include the transitions of the uncompensated defects localized at levels above the valence band. The second knee happens in the vicinity of the band gap energy and corresponds to the onset of the band-to-band carrier excitation. The peak seen in $1.2 \mathrm{eV}$ may be a noise coming from the SPV measurements. However the experiments were reproducible. Thus, it can be a shallow donor level in the CIGSe absorber layer in close level to the minimum of conduction band.

The very small values of the SPV signal $(2-7 \mu \mathrm{V})$ were suggested to arise from the high recombination rate of carriers at the surface [7]. However, the strong electric field at the interface of the absorber/window layer of the cell (normally $\sim 1 \mu \mathrm{V} / \mathrm{cm}$ ) can collect the carriers at the space charge region. Several slopes in the SPV signal indicate the variation of the CIGSe band gap, the presence of surface states, and acceptor/donor defects (deep/shallow).

At photon energies close to $E_{g}$, the SPV signal should increase rapidly up to a saturation value and $E_{g}$ should be determined by the energy position of the knee. Sometimes, as in the present case, a peak rather than a knee is observed due to electron-hole recombination at the surface which strongly reduces the SPV signal for $E<E_{g}[10]$. The main peak can be related to the energy gap value $E_{g}$ of the other compounds. Thus, at photon energies close to $E_{g}$, electronhole pairs are generated in the near-surface layer and mainly recombine there through the available high density of surface states and thus the resulting SPV signal to decrease. At $h v>E_{g}$, the signal starts decreasing due to electron-hole recombination at the surface, and then it increases again to a maximum at $1.3 \mathrm{eV}$. The measured $E_{g}$ in our experiments is in agreement with the reported values in [6]. The SPV spectrum normalized to its maximum value is reported in Figure 2. Grating A with 300 lines/ $\mu \mathrm{m}$ allowed higher energy $(2.6 \mathrm{eV})$ in the spectrum to be measured. Now, the increased slope of the SPV signal represents the band gap energy at which the photon energies can excite the charge carriers and change the surface band bending. The excitation occurs after $2.7 \mathrm{eV}$ and a sharp knee is seen until $3 \mathrm{eV}$. A linear slope extraction gives $E_{g}=2.6 \mathrm{eV}$ relevant to a defect level in $\mathrm{ZnO}$.

In Figure 2 at higher energies there are still some slopes at $E>2.8 \mathrm{eV}$ which are bigger than the band gap of the device layers. These features are probably relevant to the transitions at the interface where the electrons from the conduction band in the CIGSe layer recombine with the holes at the valence band of CIGSe layer. In this work we could not observe a peak at $2.2 \mathrm{eV}$ relevant to $\mathrm{In}_{2} \mathrm{~S}_{3}$ layer which means that this layer was thin enough and transparent to the respective photons. Similarly, they related the peaks for energies above $2.8 \mathrm{eV}$ to the Xe lamp used for illuminating the device. In some investigations with SPV techniques, such anomalies were attributed to the transitions from the defect levels to the subband gap levels of the adjacent layers [7]. They rather concluded that when the SPV spectrum, detected from illuminated CIGSe/ $\operatorname{In}_{2} \mathrm{~S}_{3}$, started to rise at photon energies above $1.2 \mathrm{eV}$ the formation of the CIGSe/ $\mathrm{In}_{2} \mathrm{~S}_{3}(\mathrm{Cl})$ interface was very influential on the separation of charge carriers photogenerated in the $\mathrm{In}_{2} \mathrm{~S}_{3}$ layer. This effect concerns the existence of acceptor/donor levels in the respective $n$-type

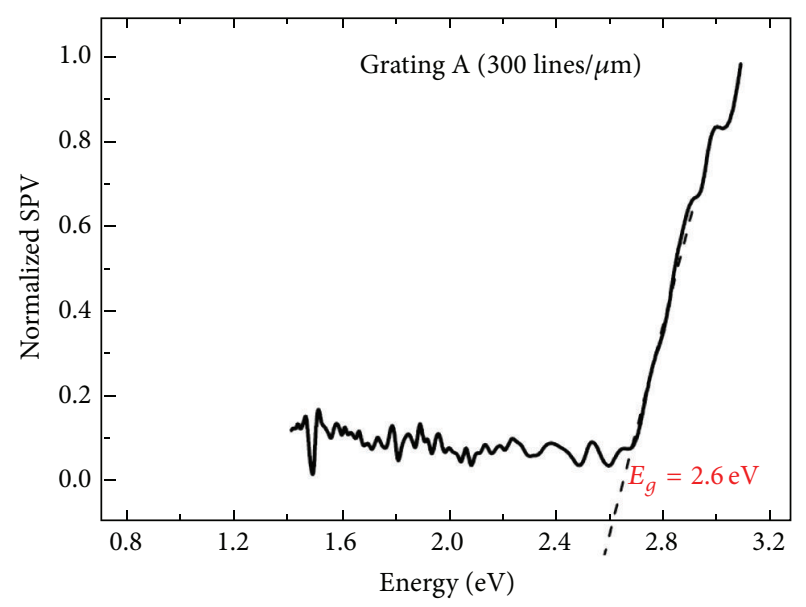

FIGURE 2: Normalized SPV spectrum versus photon energy impinging through grating A (300 lines/ $\mu \mathrm{m})$.

and $p$-type layers. Such transitions are not far from the expectation because the treatment and postgrowth annealing induce many different defects, states, and complexes at both surface and bulk of the materials. Interface states play an essential role in the origin of the SPV signals. Their effect can be rather investigated by photoluminescence measurements [11]. The concentration of holes at each layer can have a similar effect localized at interface states. Since the SPV signal amplitude is related to the concentration of the excited carriers, one could expect the signal to be higher if the device goes under illumination. Thus the back surface could have bigger signals than the interface ones due to the lower recombination rate at the back surface. It is known that the recombination rate at the junction of a $p n$ structure has the maximum rate. In order to obtain an insight into the source of defects and interdiffusion of the materials into the adjacent layers as well as the surface roughness and homogeneity of the film, the AFM analysis was performed on the same sample.

3.2. AFM Analysis. Dittrich et al. have applied the SPV technique to CIGSe thin film solar cells with two different $\mathrm{CdS}$ and $\mathrm{In}_{2} \mathrm{~S}_{3}$ buffer layers [9]. They obtained higher SPV signals for the device with $\operatorname{In}_{2} S_{3}$. However, they reported that the deposition of $\mathrm{ZnO}$ can significantly change the amplitude of the SPV signal. They measured the SPV signal after $\mathrm{ZnO}$ deposition which was lower than the one detected for the cell with CdS. This observable change was attributed to the variation in the absorption range relevant to defect states with levels below the band gap of $\operatorname{In}_{2} S_{3}\left(E_{g}=2.6 \mathrm{eV}\right)$. Unfortunately, the morphology of the device was not performed to obtain a clearer idea about the distribution of defects through the film and/or the grain size and the boundary. Photoluminescence measurements or atomic force microscopy could be applied correspondingly.

Atomic force microscopy was used to map the surface morphology and phase inhomogeneities of the same device. The grading ratio of the In and Ga concentrations can spatially vary the surface energy due to differences in the chemical composition. Thus, a phase image can represent 


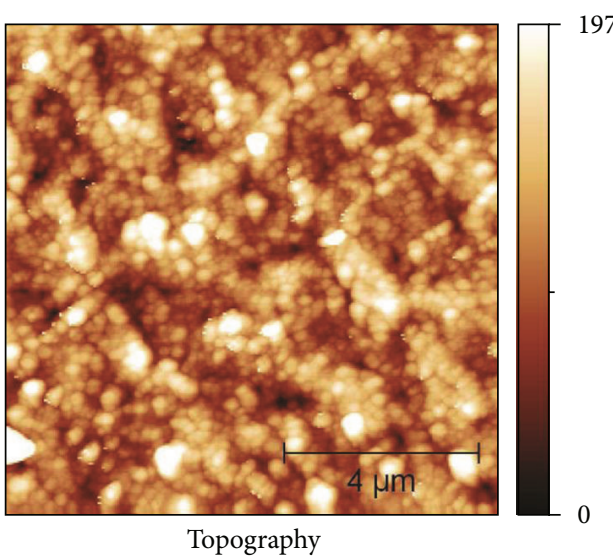

(a)

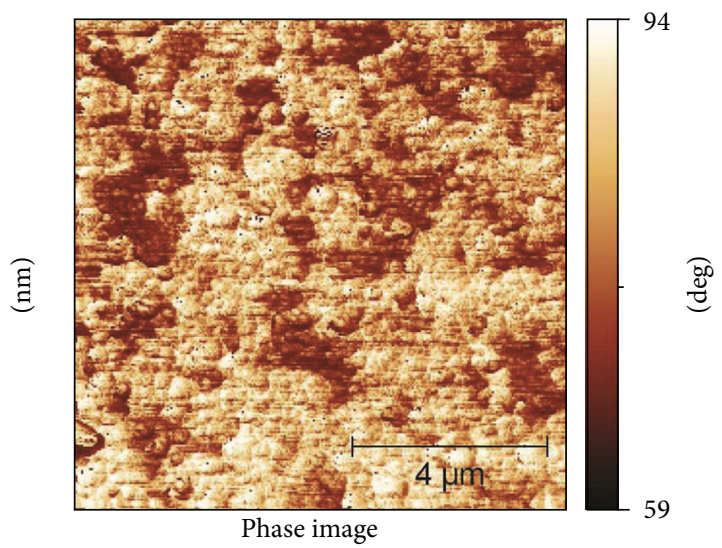

(b)

FIgURE 3: Topography and phase images of the CIGSe surface showing the roughness and grain size distribution.

the compositional surface inhomogeneity [11]. Figure 3 shows the topographic images obtained in AFM semicontact mode surface in the dark at low positive sample bias. The estimated average root mean square (rms) roughness is about $37.8 \mathrm{~nm}$ for a $400^{\circ} \mathrm{C}$ annealed film. The grains have almost uniform sizes of about $1 \mu \mathrm{m}$. The morphology shows the presence of granular grains typical of three-stage deposition and treatment solutions $\left(\mathrm{CdCl}_{2}\right.$ or deionized water) as white spots. Figure 3 displays also the phase image of the same area. The variation of grains and grain boundaries (GB) are due to differences in crystallographic orientation, contaminants, and film quality. The pixels in the two images have a corresponding relationship.

The surface height in dark red parts is $100 \mathrm{~nm}$ and in lighter ones is lower than $190 \mathrm{~nm}$ with 94 -degree phase. The phase image is a signature of inhomogeneity in the composition of the sample surface; this image can be attributed to In segregation at surface defect states [8]. Indium would more likely segregate, as its surface diffusivity is higher than that of the other chemical components. The surface of the cell looks slightly rough $(\mathrm{rms}=37.8 \mathrm{~nm})$ but almost smooth with no cracks. This conclusion is attributed to the presence of an $\operatorname{In}_{2} \mathrm{~S}_{3}$ buffer layer. Clearly the growth conditions (e.g., annealing) have a strong influence on the surface topography. The improvement in crystallinity occurs by the recrystallization of the layers under annealing at high temperature. Comparing the rms of the treated and as-deposited films, it can be deduced that the treated surface at $400^{\circ} \mathrm{C}$ is smoother than that of the as-deposited films annealed at $200^{\circ} \mathrm{C}$. A smoother film reduces the light trapping at the surface and the number of interface states at CIGSe/ $\operatorname{In}_{2} \mathrm{~S}_{3}$. The films show (112) oriented peaks with slightly random orientation and the grain average size is about $1 \mu \mathrm{m}$. The three-stage process reduces the orientation of the nucleation but the grain sizes are smaller than those of the four-source elemental evaporation. Insertion of an $\operatorname{In}_{2} \mathrm{~S}_{3}$ layer improved the surface roughness of the film comparing with the same sample and AFM results that are reported in [5]. Probably, the intermix of the layers from CIGSe to $\mathrm{In}_{2} \mathrm{~S}_{3}$ or interdiffusion of $\mathrm{Cu}$ into $\operatorname{In}_{2} \mathrm{~S}_{3}\left(\mathrm{In}_{2} \mathrm{~S}_{3}: \mathrm{Cu}\right)$ and vice versa ceased due to this interface layer. Rather analyses are required to understand the interdiffusion and grain nucleation mechanism in thin films solar cells [12-14].

\section{Summary}

In summary, a graded band gap chalcopyrite CIGSe solar cell has been investigated by the surface photovoltage spectroscopy and atomic force microscopy. The band gap of a thin film solar cell based on CIGSe can be graded through a coevaporation stage. This is a conventional way to enhance light absorption as well as a better carrier extraction in a CIGSe solar cell. The band gap grading range is deduced by the extrapolation of the SPV signal slopes to the photon energy axis giving $E_{g}=1.1-1.2 / 1.3 \mathrm{eV}$ which is acceptable for the In concentration against $\mathrm{Ga}$ of about 0.3 . The $\mathrm{ZnO}$ shows a $2.6 \mathrm{eV}$ band gap in agreement with the conventional reports on that. The SPV analysis can lead to understanding the effect of surface states and/or uncompensated defect states present in the CIGSe or $\mathrm{ZnO}$ layer as well as the interdiffusion of $\mathrm{Cu}$ into $\mathrm{In}_{2} \mathrm{~S}_{3}$ layer. The photoluminescence measurements can also assist in understanding the levels of transition and the band gap grading induced by changes in the material components. However, the levels of subband gap transitions and defects can also be reviewed by the nondestructive SPV technique in room temperature. Such studies are extremely subject of debate among photovoltaic specialists and the effectiveness of any method to understand a specific phenomenon can be comparable and meaningful. The SPV signal observed at $1.3 \mathrm{eV}$ is attributed to signature of $\operatorname{In}_{2} \mathrm{~S}_{3}: \mathrm{Cu}$ which is formed because of the $\mathrm{Cu}$ diffusion from CIGSe into $\mathrm{In}_{2} \mathrm{~S}_{3}$. Thus also the band gap of $\mathrm{In}_{2} \mathrm{~S}_{3}$ is much smaller than $2 \mathrm{eV}$. The second SPV peak at $2.6 \mathrm{eV}$ is attributed to metastabilities in CIGSe cells and could be also due to a defect level in $\mathrm{ZnO}$. The AFM measurements yield information on the orientation of the atomic nucleation and material homogeneity. The grains are very oriented and the average size is almost uniform (less than $1 \mu \mathrm{m}$ ). The topography shows a uniform diffusion of 
$\mathrm{In}_{2} \mathrm{~S}_{3}$ or deionized water into the absorber layer after the treatment and annealing. The surface of the cell looks slightly rough $(\mathrm{rms}=37.8 \mathrm{~nm})$ but almost smooth with no cracks.

\section{Conflict of Interests}

The authors declare that there is no conflict of interests regarding the publication of this paper.

\section{Acknowledgments}

The authors thank Professor N. Barreau from the Université de Nantes, Nantes, France, for providing the sample. The authors highly appreciate Professor A. Cavallini, Dr. A. Minj, and S. Pandey from the Department of Physics, University of Bologna, Bologna, Italy, for their collaboration and valuable discussions. This work was partially funded by Intel Doctoral Student Honor Award, 2013.

\section{References}

[1] S. Pandey, D. Cavalcoli, and A. Cavallini, "Band bowing and Si donor levels in InGaN layers investigated by surface photo voltage spectroscopy," Applied Physics Letters, vol. 102, no. 14, Article ID 142101, 2013.

[2] N. E. Gorji, U. Reggiani, and L. Sandrolini, "Graded band gap CIGS solar cells considering the valence band widening," in Proceedings of the 38th IEEE Photovoltaic Specialists Conference, pp. 906-908, 2012.

[3] L. Kronik, B. Mishori, E. Fefer, Y. Shapira, and W. Riedl, "Quality control and characterization of $\mathrm{Cu}(\mathrm{In}, \mathrm{Ga}) \mathrm{Se}_{2}$-based thin-film solar cells by surface photovoltage spectroscopy," Solar Energy Materials and Solar Cells, vol. 51, no. 1, pp. 21-34, 1998.

[4] B. Fraboni, D. Cavalcoli, A. Cavallini, and P. Fochuk, "Electrical activity of deep traps in high resistivity CdTe: spectroscopic characterization," Journal of Applied Physics, vol. 105, no. 7, Article ID 073705, 2009.

[5] Y.-K. Liao, S.-Y. Kuo, M.-Y. Hsieh et al., "A look into the origin of shunt leakage current of $\mathrm{Cu}(\mathrm{In}, \mathrm{Ga}) \mathrm{Se}_{2}$ solar cells via experimental and simulation methods," Solar Energy Materials and Solar Cells, vol. 117, pp. 145-151, 2013.

[6] F. Jacob, N. Barreau, S. Gall, and J. Kessler, "Performance of $\mathrm{CuIn}_{1-x} \mathrm{Ga}_{x} \mathrm{Se}_{2}$ /(PVD) $\mathrm{In}_{2} \mathrm{~S}_{3}$ solar cells versus gallium content," Thin Solid Films, vol. 515, no. 15, pp. 6028-6031, 2007.

[7] S. Pandey, D. Cavalcoli, A. Minj et al., "Electronic transitions and fermi edge singularity in polar heterostructures studied by absorption and emission spectroscopy," Journal of Applied Physics, vol. 112, no. 12, Article ID 123721, 2012.

[8] H. Mönig, Y. Smith, R. Caballero, C. Kaufmann, M. Lux-Steiner, and S. Sadewasser, "Direct evidence for a reduced density of deep level defects at grain boundaries of $\mathrm{Cu}(\mathrm{In}, \mathrm{Ga}) \mathrm{Se}_{2}$ thin films," Physical Review Letters, vol. 105, no. 11, Article ID 116802, 2010.

[9] T. Dittrich, A. Gonzáles, T. Rissom et al., "Comparative study of $\mathrm{Cu}(\mathrm{In}, \mathrm{Ga}) \mathrm{Se}_{2} / \mathrm{CdS}$ and $\mathrm{Cu}(\mathrm{In}, \mathrm{Ga}) \mathrm{Se}_{2} / \mathrm{In}_{2} \mathrm{~S}_{3}$ systems by surface photovoltage techniques," Thin Solid Films, vol. 535, no. 1, pp. 357-361, 2013.

[10] D. Cavalcoli, B. Fraboni, and A. Cavallini, "Surface photovoltage spectroscopy analyses of $\mathrm{Cd}_{1-x} \mathrm{Zn}_{x}$ Te," Journal of Applied Physics, vol. 103, no. 4, Article ID 043713, 2008.
[11] A. Darga, W. Favre, M. Fruzzetti et al., "Study of the electronic properties of wide band gap CIGSe solar cells: influence of copper off-stoichiometry," Journal of Non-Crystalline Solids, vol. 358, no. 17, pp. 2428-2430, 2012.

[12] N. E. Gorji, U. Reggiani, and L. Sandrolini, "A simple model for the photocurrent density of a graded band gap CIGS thin film solar cell," Solar Energy, vol. 86, no. 3, pp. 920-925, 2012.

[13] A. Minj, D. Cavalcoli, and A. Cavallini, "Investigation of the properties of In-related alloys by AFM," Physica Status Solidi C, vol. 9, no. 3-4, pp. 982-985, 2012.

[14] A. Minj, D. Cavalcoli, and A. Cavallini, "Structural and local electrical properties of AlInN/AlN/GaN heterostructures," Physica B: Condensed Matter, vol. 407, no. 15, pp. 2838-2840, 2012. 

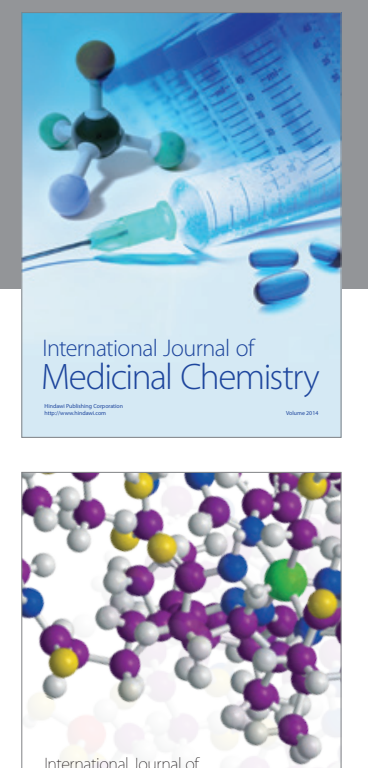

\section{Carbohydrate} Chemistry

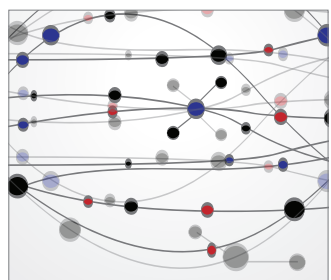

The Scientific World Journal
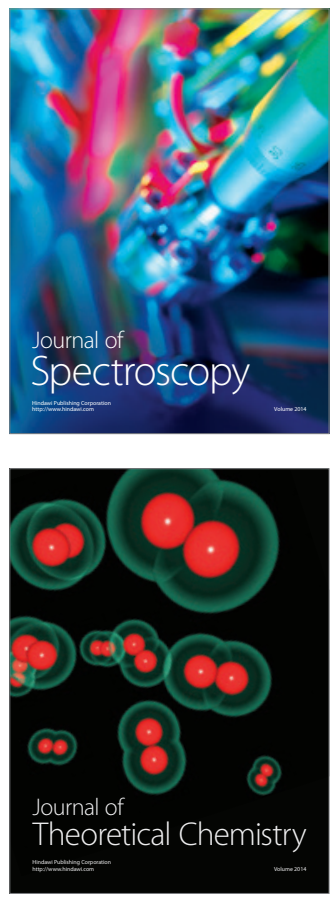
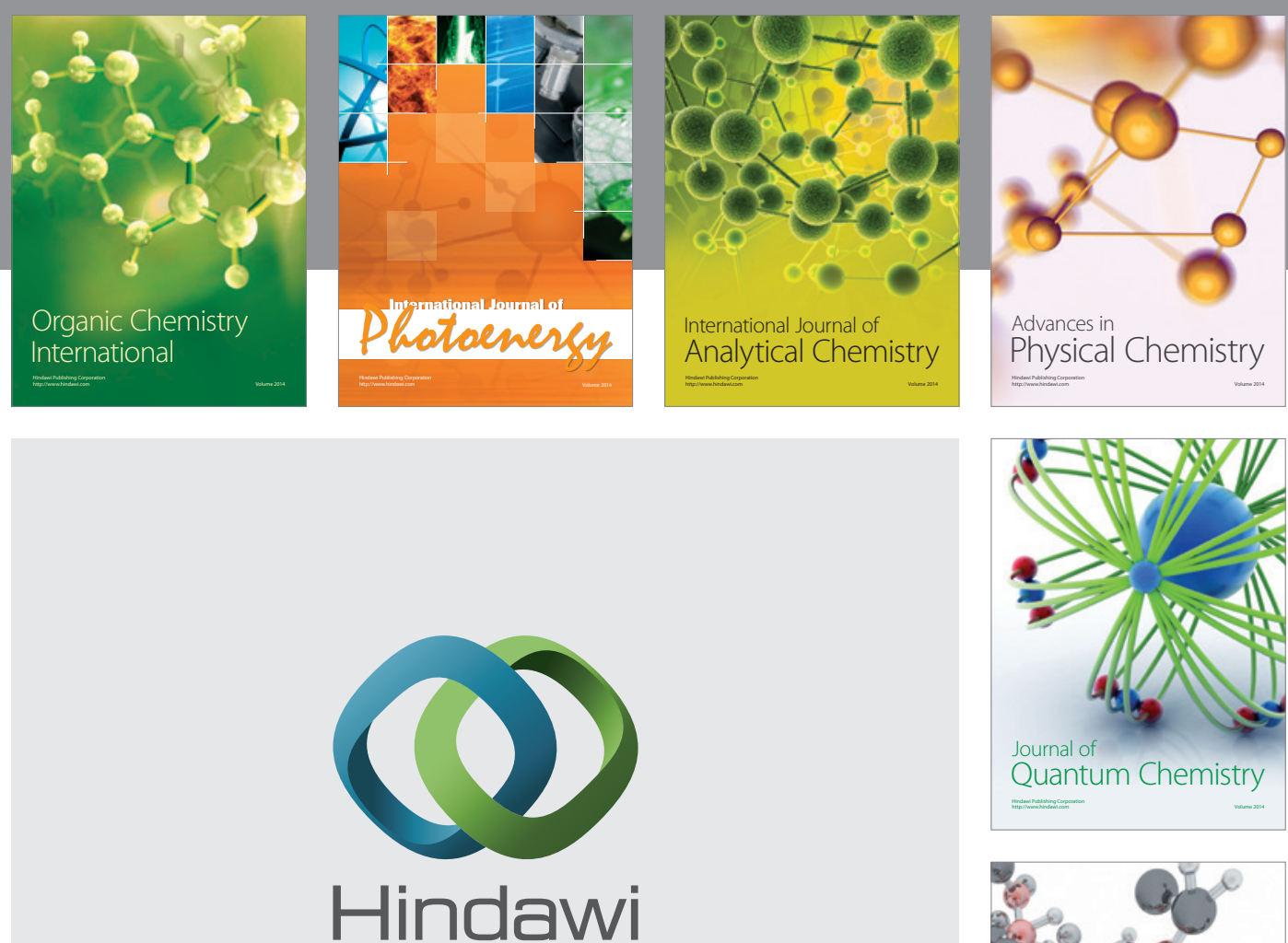

Submit your manuscripts at

http://www.hindawi.com

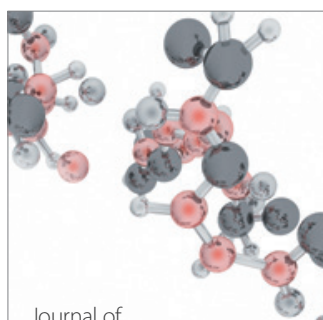

Analytical Methods

in Chemistry

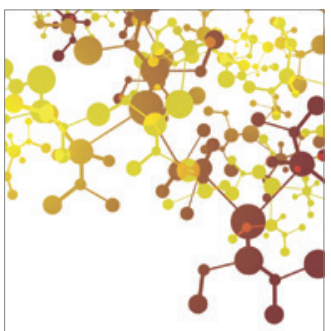

Journal of

Applied Chemistry

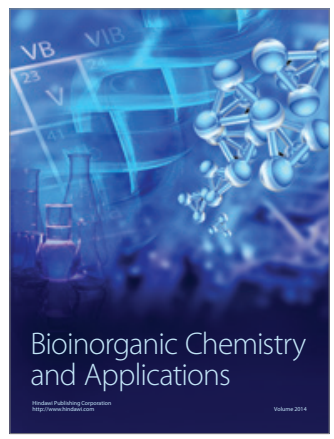

Inorganic Chemistry
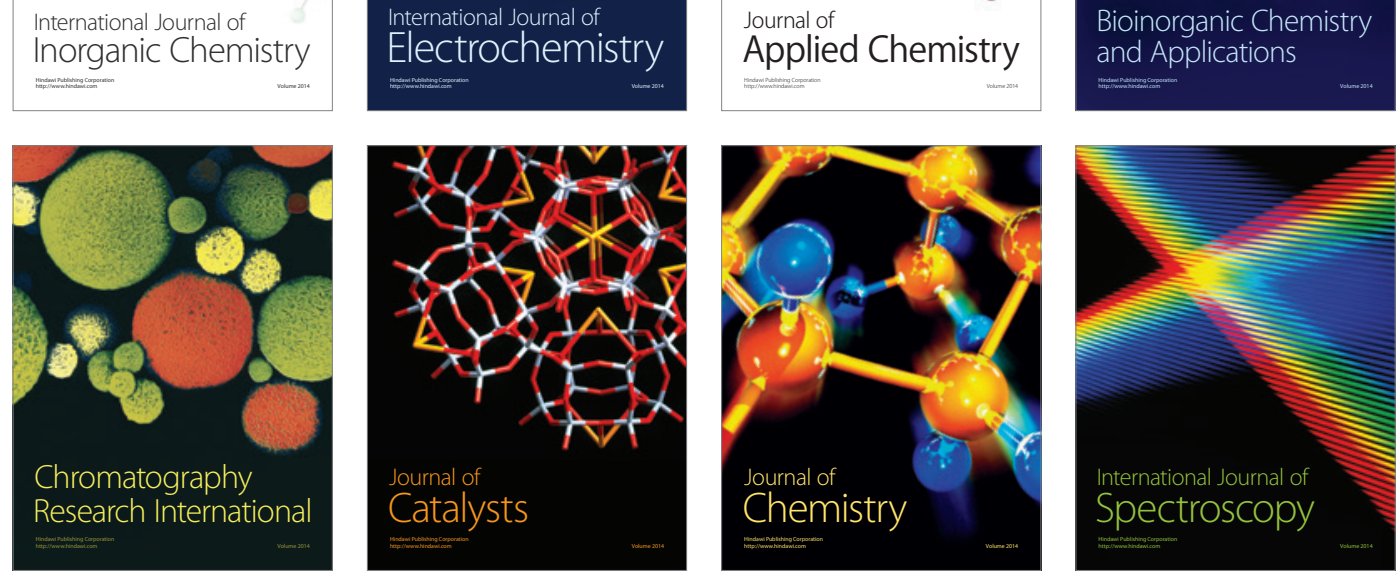